\title{
Management of Patients with Differentiated Thyroid Carcinoma- SNMB Guidelines
}

Sadia Sultana ${ }^{1}$, Nurun Nahar ${ }^{1}$, Fatima Begum ${ }^{2}$, Faridul Alam ${ }^{3}$, Mizanul Hasan ${ }^{1}$, Raihan Hussain ${ }^{1}$, Mahmubul Haque ${ }^{4}$, Faria Nasreen ${ }^{2}$, Mohafizul Haque Khan ${ }^{5}$, Lutfun Nisa ${ }^{6}$, Fauzia Moslem ${ }^{7}$, Shahana Afroz $^{8}$, Kamila Afroj Quadir', Ferdoushi Begum ${ }^{2}$, M. A. Karim ${ }^{9}$

${ }^{1}$ Professor, NINMAS, BAEC, ${ }^{2}$ Associate Professor, NINMAS, BAEC, ${ }^{3}$ Director, BIHS, ${ }^{4}$ Director, INMAS, Mitford, BAEC, ${ }^{5}$ Director, NINMAS, BAEC, ${ }^{6}$ Former Professor, NINMAS, BAEC, ${ }^{7}$ Head, Dept. of Radiology and Imaging, BIHS, ${ }^{8}$ Member, Bangladesh Atomic Energy Regulatory Authority, ${ }^{9}$ Former Chairman, BAEC

Scope of Nuclear Medicine in the management of thyroid carcinoma:

Among thyroid carcinomas, differentiated thyroid carcinoma (DTC) can be treated with radioactive iodine (RAI) ${ }^{131}$ I due to iodine uptake property of malignant follicular cells. (For classification of thyroid malignancy see annexure 1). With this idea Seidlin et al. started using ${ }^{131} \mathrm{I}$ in the treatment of metastatic DTC in 1943 and published first article in 1946.

In Bangladesh RAI is being used for treatment of DTC since 1980. Society of Nuclear Medicine, Bangladesh (SNMB) formulated the first official protocol for management of patients with differentiated thyroid carcinoma (DTC) in 2001 and accordingly a guideline was published in Bangladesh Journal of Nuclear Medicine (BJNM) in 2002. Since then a large amount of information has become available according to which we have made certain modifications in management of patients with DTC in National Institute of Nuclear Medicine \& Allied Sciences (NINMAS). Still certain fields required incorporation of modification. In a word, up-gradation of the previous protocol has become rational claims of time for current medical practice.

With this idea, a number of meeting and discussions were held in National Institute of Nuclear Medicine \& Allied Sciences (NINMAS), and a protocol was proposed which was presented in $19^{\text {th }}$ annual conference of SNMB on $27^{\text {th }}$ March 2014 in Cox's Bazar to seek opinion of nuclear medicine specialists. With their suggestions a modified protocol was presented in a seminar organized by SNMB in cooperation of NINMAS on $12^{\text {th }}$ June 2014 where specialists from other disciplines like ENT, Pathology and Endocrinology were present. After thorough discussions a guideline was drafted which was again presented on 26 February 2015 in seminar organized by SNMB \& NINMAS in Dhaka for final approval. The present guideline is output of that.

Besides discussions to prepare this guideline many published guidelines, relevant articles published in authentic international journals and experiences of NINMAS were considered.

\section{Management of DTC}

- Total thyroidectomy is the mainstay of treatment. (Rationale of total thyroidectomy Annexure 2; definition of thyroidectomy Annexure 3.)

- Lymph nodes in lateral neck (level II- V), anterior mediastinal (level VI) and rarely level I may also be involved in thyroid cancer (Annexure 4).

- Lymph node dissection should accompany total thyroidectomy (TT) or near total thyroidectomy (NTT), where necessary (Annexure 5)

- Radioactive iodine (RAI) ${ }^{131}$ I (Properties of ${ }^{131}$ I Annexure 6) is administered as adjuvant therapy

- to reduce risk of recurrence and

- to facilitate future cancer surveillance.

- RAI ablation should be completed within 46 weeks of thyroidectomy 


\section{Goal of RAI ablation}

- As adjuvant therapy-

- To eliminate thyroid remnants to increase sensitivity of thyroglobulin (Tg) test and diagnostic whole body scan (DxWBS) to detect persistent or recurrent disease

- Ablation allows sensitive post-therapy whole body scan (RxWBS) that may detect occult metastases and

- To treat micro-metastases, known lymph nodes and distant metastases.

Knowledge about TNM classification, tumor grading and staging is very important for decision making

\section{TNM classification}

According to American Joint Committee on Cancer (AJCC) (2002)

\section{Primary tumor (T)}

TX- Primary tumor cannot be assessed

T0- No evidence of primary tumor

T1- Tumor $2 \mathrm{~cm}$ or less, limited in thyroid bed

T2- Tumor $>2 \mathrm{~cm}$ but $<4 \mathrm{~cm}$, limited to thyroid bed

T3- Tumor $>4 \mathrm{~cm}$, limited to thyroid bed or any tumor with minimal extra thyroidal extension (extension to extra thyroid muscle or parathyroid soft tissue)

T4a- Tumor of any size extending beyond thyroid capsule and invade subcutaneous soft tissue, larynx, trachea, esophagus or recurrent laryngeal nerve

T4b- Tumor invades prevertebral fascia or encases carotid artery or mediastinal vessels

All anaplastic tumors are T4 tumors

\section{Regional lymph nodes (N)}

Stage 4B

Regional lymph nodes are central compartment, Stage 4C lateral cervical \& upper mediastinal nodes

Stage 3

Stage 4A

NX- Regional LN cannot be assessed

N0- No regional LN metastases

N1- Regional LN metastases

N1a- Metastases to central compartment LN or level VI nodes

N1b- Metastases to unilateral, bilateral or contralateral cervical or superior mediastinal LN

\section{Distant Metastases (M)}

MX-Distant metastases cannot be assessed

M0- No distant metastases

M1- Distant metastases

\section{Grading of thyroid carcinoma}

According to American Joint Committee on Cancer (AJCC) (2002)

GX-Grade cannot be assessed (Undetermined)

G1- well differentiated (Low grade)

G2- moderately differentiated (Intermediate grade)

G3- poorly differentiated (High grade)

G4- Undifferentiated (High grade)

\section{Staging of thyroid carcinoma}

According to American Joint Committee on Cancer (AJCC) (2002)

\section{Under 45yrs}

Stage 1 any T, any N, M0

Stage 2 any T, any N, M1 


\section{Indications of RAI ablation}

- RAI ablation is indicated for patients in high \& intermediate risk groups.

- Low risk group patients - RAI ablation should be given with low activities like 30$50 \mathrm{mCi}$.

- Papillary micro-carcinoma - RAI ablation may be avoided in patients who are compliant to regular follow-up. Otherwise should be treated with low activities of RAI.

- Follicular thyroid carcinoma whether minimally invasive or widely invasivemust be treated with RAI.

American Thyroid Association (ATA) definition of high, intermediate and low risk patients:

- High risk patients

- Age $>45$ years with tumor size $>4 \mathrm{~cm}$,

- Tumor of any size, any age with gross extra thyroidal extension and

- Distant metastases

- Low risk patients

- Tumor $<1 \mathrm{~cm}$ without aggressive histology and

- No microscopic tumor invasion

- Intermediate risk patients

- Tumor 1- $4 \mathrm{~cm}$ confined to the thyroid gland,

- Tumor with aggressive histology,

- Microscopic tumor invasion and

- Lymph node metastases

\section{Patient preparation}

During first visit (preferably immediately after discharge following surgery) patient's medical records should be checked for

- Discharge certificate,

- Histopathology report,
- Preoperative neck/ thyroid ultrasonography (US) report

- Preoperative S. Creatinine level, x-ray chest, CT scan and other investigations findings, if any and

- List of medication- Thyroxine \& Calcium supplementation.

Risk stratification and Patient selection should be done according to

1) Surgical findings:

i) Details of the operation- structures operated

ii) Macroscopic capsular invasion

iii) Extrathyroidal extension

iv) Completeness of resection

v) Lymph node dissection

2) Histopathology reports:

i) Tumor size,

ii) Tumor histology including tumor sub-type,

iii) Tumor grade- well, moderately, poorly differentiated or undifferentiated,

iv) Capsular invasion,

v) Lymph-vascular invasion,

vi) Perineural invasion,

vii) Surgical margin, and

viii) Lymph node status, if any.

\section{Prerequisites of RAI ablation}

For successful ablation TSH should be $>30 \mathrm{mIU} / \mathrm{L}$ after total thyroidectomy (TT) or near total thyroidectomy (NTT).

- Postoperative evaluation is done 2 weeks after TT or NTT keeping patient without LThyroxin by

- Serum TSH,

- RAIU \& ${ }^{99 \mathrm{~m}}$ Tc thyroid scan,

- US of neck,

- Serum calcium \& parathormone (PTH) level,

- CBC, serum Creatinine, if not done previously and 
- Serum $\mathrm{Tg} \&$ anti $\mathrm{Tg} \mathrm{Ab}$ are checked on the day of RAI administration.

- In case of large remnant tissue- RAIU >5$10 \%$ with large remnant tissue in thyroid bed on ${ }^{99 \mathrm{~m}} \mathrm{Tc}$ scan and $\mathrm{TSH}$ not raised up to required level, further surgery is recommended.

- If further surgery is refused due to adhesionrepeated low activity therapies with 30-50 $\mathrm{mCi}$ of ${ }^{131} \mathrm{I}$ at 3-4 months interval should be considered.

- In case of cervical lymphadenopathy- neck dissection is mandatory.

- Remnant ablation can be done following rhTSH (Thyrogen) stimulation keeping on LThyroxin.

- Following things should be discussed with patients and relatives:

- Rationale, procedure, and side-effects of RAI therapy,

- Isolation and radioprotection during stay in the hospital \& after discharge and

- Mode of future follow-up.

- Written informed consent is to be taken before administration of RAI.

- Patient should be in low iodine diet $(<50$ $\mu \mathrm{gm} / \mathrm{d}$ of dietary iodine) for 2 weeks before and 1-2 days after ${ }^{131} \mathrm{I}$ therapy (Annexure 7).

- In cases of radiographic contrast injection (e.g. contrast CT) ${ }^{131}$ I therapy should be postponed for 2-3 months.

- Pregnancy and lactation should be excluded in case of female patients.

- In case of lactating mother- RAI therapy should be given 6-8 weeks after cessation of breast-feeding.

\section{Procedure}

- Patients should be hospitalized for ${ }^{131} \mathrm{I}$ therapy.

- $30-100 \mathrm{mCi}$ is administered for remnant ablation.

- Higher activities (100-200 mCi) are selected for lymph node and distant metastases.

- In pediatric patients activities of ${ }^{131}$ I should be adjusted according to body weight or surface area.

- To avoid vomiting following RAI administration

- Give anti-emetics before mid-day meal (as RAI usually administered in afternoon) and

- No food should be allowed for at least 2 hours before and 1 hour after RAI administration

- Supportive medicines like anxiolytic, antiulcerent, NSAID should be advised.

- Vitamin C, lemon candies and other sialagogues should be started after 24 hours.

- Start L- thyroxine (LT4) on $2^{\text {nd }}$ or $3^{\text {rd }}$ posttherapy day (PTD) in incremental dose.

- Calcium supplementation with vitamin D is given where necessary.

- Calcitriol should be given in selective cases.

- Purgatives are given on the $2^{\text {nd }}$ or $3^{\text {rd }}$ posttherapy day.

- Patients are advised to drink plenty of liquid from $1^{\text {st }}$ post-therapy day and empty bladder frequently.

\section{Isolation and radioprotection}

- No visitor should be allowed during first 24 hours.

- No contact with children and pregnant women for 10-15 days.

- Adults may be allowed for short period of time keeping sufficient distance $(>1$ meter) (Annexure 8).

- Duration of isolation can be calculated using age and kidney factor (Annexure 9). 


\section{Release from isolation}

- Patients can be released from isolation when survey meter count is $20-25 \mu \mathrm{Sv} / \mathrm{h}$ at 1 meter distance.

- Tumor uptake is confirmed by post-therapy whole body scan (RxWBS) 2-10 days after therapeutic dose.

- In NINMAS, RxWBS is usually done on $5^{\text {th }}$ PTD and patients are released from isolation with survey meter count is $<10$ $\mu \mathrm{Sv} / \mathrm{h}$ at $1 \mathrm{~m}$ distance.

\section{Instructions during discharge}

- To reduce external exposure to others:

- Limit time spend in public place.

- Do not travel by mass transportation.

- Delay returning to work if workplace or office is shared.

- Avoid contact with children and pregnant women.

- Sleep in separate bed.

- Use separate bathroom, if possible.

- Flush toilet at least twice after each use and flush used facial tissue down the toilet.

- To minimize transfer of ${ }^{131} \mathrm{I}$ or internal exposure:

- Avoid mouth to mouth contact.

- Do not share item that comes in contact with mouth or saliva (eating utensils, water bottles).

- Use separate towels and washcloths.

- Wash utensils and plates separately.

- Wash hands thoroughly after going to toilet.

- Do not cook and feed children.

- Avoid pregnancy for one year and fathering a child for 6 months following RAI therapy

Care about contaminated items (please see Annexure 10).
Pathological and clinical staging after RAI ablation

- Benefit of staging:

- Staging at this stage allows risk stratification of individual patient which in turn will dictate the frequency and type of follow-up.

- Staging of DTC is based on the pathological assessment with addition of information derived from the RxWBS.

\section{- Low risk patients:}

- No tumor invasion in loco-regional tissue or structure,

- No vascular invasion,

- All microscopic tumor has been resected,

- Tumor does not have aggressive histology (e.g. tall cell, columnar cell, insular), and

- No uptake outside thyroid bed on RxWBS.

\section{- Intermediate risk patients:}

- Microscopic invasion of tumor into the peri-thyroidal soft tissues at the initial surgery,

- Tumor with aggressive histology,

- Vascular invasion,

- Cervical lymph node metastases, and

- ${ }^{131}$ I uptake outside the thyroid bed on the RxWBS.

\section{- High-risk patients:}

- Macroscopic tumor invasion,

- Incomplete tumor resection,

- Distant metastases, and

- Thyroglobulinemia irrespective of findings on RxWBS.

\section{Thyrotropin-suppressive therapy}

- Rationale \& recommendation:

- To correct hypothyroidism and

- To inhibit TSH dependent growth of residual cancer cells by suppressing TSH. 
- L-thyroxine (LT4) is the drug of choice

- Suppressive dose of LT4

- Young patients- $2.0-2.2 \mu \mathrm{gm} / \mathrm{kg} / \mathrm{day}$

- Elderly patients- $1.5-1.8 \mu \mathrm{gm} / \mathrm{kg} /$ day

- Initial TSH suppression is

$-\leq 0.1 \mathrm{mIU} / \mathrm{L}$ for high $\&$ intermediate risk group

- 0.1-0.5 mIU/L for low risk group

- TSH is measured 3 months after initiation of LT4

- Dose of LT4 is adjusted according to patients TSH level by a decrease or increase of $12.5-25 \mu \mathrm{gm} /$ day at 6- 8 weeks \&

- Serum TSH is monitored again after 3 months.

\section{Follow-up after initial therapy}

- Objectives of follow up:

- To ensure that the patients receive lowest effective dose of LT4 i.e. one that provides required TSH suppression

- To discover at the earliest possible time persistent or recurrent disease

\section{First follow-up after RAI ablation}

- $\quad$ The 3-month follow-up

- $\quad$ Patient is on LT4

- Physical examination

- TSH, FT3 or FT4

- $\mathrm{Tg} \&$ anti Tg antibody (TgAb)

- Neck US (HRUS)-

- Cervical lymph node $>5-8 \mathrm{~mm}$ in the smallest diameter should go for biopsy

- Suspicious lymph nose $<5-8 \mathrm{~mm}$ in largest diameter should be subjected to regular follow up

- $\mathrm{CBC}$, serum calcium

- CXR (where necessary)
- At this stage, $\mathrm{TSH} \leq 0.1 \mathrm{mIU} / \mathrm{L}$, normal FT3 and normal or high FT4 level denote an appropriate dose of LT4.

- Patient is considered as no evidence of disease:

- complete tumor resection according to the surgeon's report,

- no uptake outside the thyroid bed on RxWBS,

- undetectable $\mathrm{Tg}$ levels $(<1 \mathrm{ng} / \mathrm{ml}$ during LT4 treatment) and

- no abnormality on neck US.

- In case of persistent disease further therapy is delayed for 6-12 months.

\section{Second follow-up}

- $\quad$ The 6-month follow-up (on LT4)

For patient without evidence of disease at 3month, the 6 -month follow-up consists of

- $\quad$ Serum TSH

- Serum Tg and anti Tg antibody measurement

- Neck US (HRUS)-

- Cervical lymph node $>5-8 \mathrm{~mm}$ in the smallest diameter should go for biopsy

- Suspicious lymph nose $<5-8 \mathrm{~mm}$ in largest diameter should be subjected to regular follow up

- $\quad$ CBC, Serum calcium

\section{Third follow-up}

- The 12-month follow-up consists of

- Measurement of stimulated $\mathrm{Tg}$ and anti Tg antibody following LT4 withdrawal for 3 weeks \&

- DxWBS with 3- 5 mCi of ${ }^{131}$ I which is must for intermediate \& high-risk patients. May be avoided in low-risk patients.

- $\quad$ BBC \& serum calcium 


\section{Criteria of successful RAI ablation}

- Negative DxWBS,

- Absence of detectable TSH-stimulated Tg $(<1 \mathrm{ng} / \mathrm{ml})$ with negative anti Tg antibody,

- Absence of suspicious findings on neck US.

\section{Subsequent follow-up}

- With undetectable Tg, negative DxWBS and normal US findings at 6 to $12-$ month follow-up, the risk of recurrence is $<0.5 \%$.

- Reduced dose of LT4 to allow TSH concentration of $0.1-0.5 \mathrm{mIU} / \mathrm{L}$.

- Follow up patients twice yearly with TSH, $\mathrm{Tg}$, anti $\mathrm{Tg}$ antibody and US of neck keeping patient on LT4.

- Further DxWBS should be done whenever necessary.

\section{Further RAI therapy}

- Detectable stimulated Tg should be followed up and a rising $\mathrm{Tg}$ should be considered as persistent or recurrent disease and should be treated with high activities of ${ }^{131} \mathrm{I}$.

- Positive DxWBS needs further RAI therapy with higher activities.

\section{Management of loco-regional metastases}

- Surgery is favored for loco-regional recurrence (cervical LNs and soft tissue tumor in the neck) when distant metastasis is absent. Surgery should be followed by ${ }^{131}$ I therapy.

- For aerodigestive invasion, surgical resection followed by RAI and/or external beam radiotherapy (EBRT) is recommended.

- If surgery is not possible, EBRT followed by RAI is recommended.
- Ethanol injection is considered in cases of enlarged lymph nodes $(<2 \mathrm{~cm})$ where operative treatment is refused and multiple RAI therapy is failed.

\section{Management of pulmonary metastases}

- Pulmonary micro-metastases- RAI therapy is recommended and it should be repeated every 6-12 months as long as disease concentrates RAI and respond clinically.

- Empiric dose 100- $200 \mathrm{mCi}$.

- Macronodular metastases may also be treated with RAI if it is iodine avid and should be repeated when objective benefit is demonstrated (i.e. decrease in the size of lesion, decreasing $\mathrm{Tg}$ ).

- Glucocorticoids should be given in case of known pulmonary metastases along with ${ }^{131}$ I to prevent radiation induced pulmonary fibrosis.

- Non-RAI avid pulmonary disease-

- No benefit with RAI.

- Not detected by DxWBS.

- May be detected by ${ }^{18}$ FDG-PET scan.

- May be benefited with chemotherapyDoxorubicin and cisplatine.

\section{Management of bone metastases}

- Complete surgical resection of isolated metastasis is recommended.

- Prognosis can be improved if ${ }^{131} \mathrm{I}$ is given at an early stage (when bone metastases are detected in RxWBS but radiograph is negative).

- DxWBS should be done at $6^{\text {th }}$ month follow-up and therapy is to be repeated if positive.

- In cases of metastasis detected in first RxWBS- $150 \mathrm{mCi}$ of ${ }^{131} \mathrm{I}$ should be given at 6 months interval until RxWBS is negative. 
- At advanced stage when radiograph shows metastases- give $150 \mathrm{mCi}$ of ${ }^{131} \mathrm{I}$ at $4-6$ months interval until RxWBS is negative.

- ${ }^{131}$ I should not be interrupted as long as metastases are ${ }^{131} \mathrm{I}$ avid, whatever the cumulative activity reached.

- Stable disease particularly when longstanding, a strategy of 'watchful waiting' can be employed.

- EBRT and glucocorticoids should be given prior to RAI therapy to minimize radiation related tumor expansion and pain exacerbation.

- Zolendronate infusions should be considered to prevent fracture.

- Ibandronate may be given in place of Zolendronate.

- Painful lesions that cannot be resected can be treated by intra-arterial embolization, radiofrequency ablation etc.

\section{Management of brain metastases}

- Complete surgical resection is first line of treatment.

- Where surgery is not possible consider EBRT.

- RAI therapy should be considered for iodine avid lesions.

- Glucocorticoid therapy is strongly recommended prior to EBRT and during RAI therapy to minimize subsequent inflammatory effects.

\section{Tg positive- DxWBS negative patients}

- ${ }^{201} \mathrm{Tl}$ or ${ }^{99 \mathrm{~m}} \mathrm{Tc}$ scan may be done to detect metastases or

- Empiric RAI therapy (100-200 mCi) may be given.

- If RxWBS is negative, no further RAI therapy should be administered.

- ${ }^{18}$ FDG-PET scan may be done to locate metastases.
- RAI negative-PET positive patients have poor survival.

- EBRT, chemotherapy may be considered at this stage.

\section{Hypocalcaemia}

- Hypocalcaemia following total thyroidectomy is quite common.

- It is due to either devascularization or accidental removal of parathyroid glands.

- It is a grave condition which needs early detection and meticulous management.

- High doses of calcium with vitamin D should be administered.

- Alfacalcidol or Calcitriol have to be added in some cases.

- In cases of severe hypocalcaemia injectable calcium is needed (should be managed by endocrinologists).

- Patients taking high doses of calcium and calcitriol following surgery should be checked for serum calcium \& PTH level and calcium supplementation should be considered accordingly.

- When calcium is administered patient should be monitored carefully:

- Serum calcium should be measured at 2/3 months interval and

- US of kidney and serum creatinine should be checked yearly.

\section{Annexure 1. Thyroid carcinoma}

\section{Types of thyroid carcinoma:}

A. Epithelial cell origin

1. Tumor with follicular cell differentiation

i) Follicular carcinoma

(1) Minimally invasive

(2) Widely invasive

(3) Hurthle cell tumor

(4) Insular carcinoma (poorly differentiated) 
ii) Papillary carcinoma

(1) Conventional

(2) Variants

(a) Follicular variant

(b) Tall cell

(c) Columnar cell

(d) Diffuse sclerosing

2. Tumors of parafollicular or C-cells

i) Medullary carcinoma

B. Non-epithelial cell origin

i) Sarcomas

ii) Malignant lymphomas

Annexure 2. Rationale of total thyroidectomy

- Bilateral cancers are common (30-85\%).

- Improves effectiveness for ${ }^{131}$ I ablation.

- Lowers dose needed for ${ }^{131}$ I ablation.

- Allows follow up with thyroglobulin (Tg) levels.

- Decreases recurrence in all groups.

- Improved survival in high risk patients.

- Decreased risk of pulmonary metastases.

\section{Annexure 3. Thyroidectomy}

Total thyroidectomy- removal of all grossly visible thyroid tissue- both lobes, isthmus and pyramidal lobe.

Near total thyroidectomy- removal of all grossly visible thyroid tissue, leaving only a small amount $(<1 \mathrm{~g})$ of tissue adjacent to recurrent laryngeal nerve near the ligament of Berry.

(TT or NTT alone is appropriate for small (T1 or T2), noninvasive, clinically node negative PTCs and most FTCs.)

Subtotal thyroidectomy- leaving $>1 \mathrm{~g}$ of tissue with the posterior capsule on the uninvolved side.

Hemithyroidectomy- complete removal of one thyroid lobe (involved lobe) including the isthmus.

(Subtotal and Hemithyroidectomy are inappropriate operations for thyroid cancer)
Annexure 4. Cervical lymph nodes

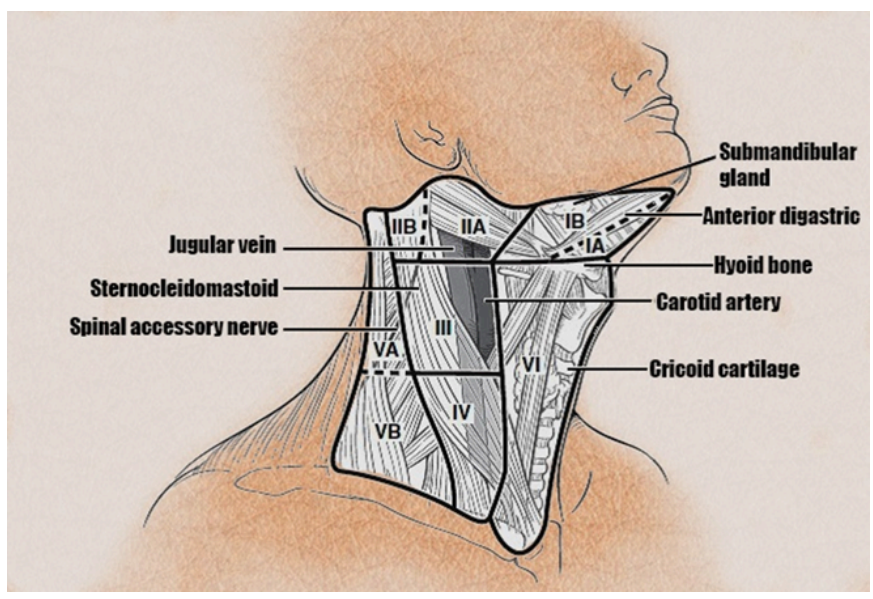

\section{Lateral compartment of neck:}

Level I- submental and submandibular nodes.

Level II- deep cervical chain nodes from the skull base to the level of the hyoid bone, which is further divided by their relationship to the spinal accessory nerve (SAN): IIa (medial) and IIb (lateral).

Level III- deep cervical chain nodes from level of the hyoid bone to the level of the cricoid cartilage.

Level IV-deep cervical chain nodes from the level of cricoid cartilage to the level of the clavicle.

II to IV neck nodes are those bordered anteriorly by carotid arteries and posteriorly by sternocleidomastoid (SCM) muscle.

Level V- posterior triangle nodes (bordered by posterior border of SCM anteriorly and anterior border of trapezius posteriorly) subdivided into $\mathrm{Va}$ and $\mathrm{Vb}$ by a plane from the inferior border of the cricoid cartilage.

\section{Central compartment of neck:}

Level VI and level VII prelaryngeal, pretrachial and paratrachial nodes from the hyoid bone superiorly to the level of the innominate artery inferiorly and to the carotid arteries laterally.

\section{Annexure 5. Lymph node dissection}

Therapeutic central-compartment (level VI) neck dissection for patients with clinically involved central or lateral neck lymph nodes should accompany TT or NTT to provide clearance of disease from the central compartment. 

Prophylactic central-compartment neck
dissection, ipsilateral or bilateral may be performed in patients with PTC with clinically uninvolved central neck lymph nodes, especially for advanced primary tumors (T3 or T4).

Selective neck dissection (SND)-any type of cervical lymphadenectomy which involves less than dissection of levels I-V with preservation of spinal accessory nerve (SAN), the internal jugular vein (IJV) and sternocleidomastoid muscle (SCM).

Radical neck dissection (RND)- removal of all the lymphatic tissue in levels I-V along with the SAN, IJV and SCM.

Modified radical neck dissection (MRND)removal of lymph nodes in levels $\mathrm{I}-\mathrm{V}$ with preservation of one or more non-lymphatic structures, e.g.

MRND type I: RND with preservation of SAN.

MRND type II: RND with preservation of SAN and IJV.

MRND type III: RND with preservation of SAN, IJV and SCM.

\section{Annexure 6. Properties of ${ }^{131} I$}

- Isotope of iodine.

- Reactor produced.

- Emits high energy gamma (364 keV).

- Decays by emitting beta particles.

- Main gamma emission is $364 \mathrm{keV}(82 \%)$ and main beta emission is $606 \mathrm{keV}(90 \%)$.

- Physical half life is 8.04 days, biological half life is 80 days in normal thyroid compartment and 12 days in extrathyroidal compartment and effective half life is 7.3 days in thyroid compartment and 8 hours in extrathyroid compartment.

- Beta particles penetrate $0.6-2.0 \mathrm{~mm}$.

- Bio-distribution-
O taken up by follicular cells of thyroid gland, salivary glands, nasal mucosa, lacrimal glands, stomach,

- Excretion via intestine and urinary tract including bladder.

\section{Annexure 7. Low-iodine diets}

Avoid followings:

- Iodized salt (non-iodized salt are available in the grocers)

- Sea food (fish, seaweed, shellfish, Kelp)

- Foods that contain agar agar

- Dairy product

- Egg yolk

- Margarine

- Chocolates containing milk

- Soybeans and soybeans product

- Iodine containing medication like amiodarone, vitamins, cough syrup etc.

\section{Annexure 8. Time spend with patient following} therapy

For 1 st 7 days-

45 min/d@3.5 feet/ 1 m

2 hrs/d@7 feet/ 2 m

7 hrs/d@10 feet/ 3 m

\section{Annexure 9. Precautions following RAI therapy for thyroid cancer}

Period of isolation may be divided into two phases:

Phase one - early, high radiation, full precaution

Phase two - late, residual radiation, minimal precaution

Should be considered according to dose of ${ }^{131} \mathrm{I}$, clinical condition of patient, age, kidney function and presence or absences of metastases

\section{Phase one}

It depends on 2 factors- age of the patient and kidney function 


\begin{tabular}{|ccc|}
\hline Age group & \multicolumn{2}{c|}{ Age/ kidney factors: } \\
\cline { 1 - 1 } & Normal kidneys & Impaired kidney \\
\hline Younger patient (<30y.) & 0.4 & up to 0.8 \\
Middle aged (30-60y) & 0.6 & up to 1.0 \\
Older patient (60+) & 0.8 & up to 1.6 \\
\hline
\end{tabular}

In phase one

Milicuries of RAI (mCi) X age/kidney factor = hours in phase 1 .

So, a young person with normal kidney function (0.4) having had $150 \mathrm{mCi}$ will be in phase one for: $0.4 \times 150=60$ hours or $2 \frac{1}{2}$ days

Similarly, an older person (60+) with impaired kidney function (1.6) having had $150 \mathrm{mCi}$ will be in phase one for: $1.6 \times 150=240$ hours or 10 days

Phase one starts immediately after treatment dose.

Phase one ends remaining radioactivity is somewhere around $5 \mathrm{mCi}$ level.

\section{Phase two}

Minimal precaution, mainly for body fluids

How long is phase two

Formula: 5days $\mathrm{x}$ age/ kidney factor $=$ days in phase two

Eg.

Young person with normal kidney: $5 \times 0.4=2$ days Older person with impaired kidney: 5 x $1.6=8$ days

Patient should be

- Careful about urine, saliva, avoid food tasting while cooking, kissing, can sleep next to an adult but avoid sleeping with children, babies and pregnant women.

- Transition from phase one to phase two is slow in cases of significant uptake due to extensive remnant thyroid tissue or metastases. In these cases precautions should be taken for distance-avoid sitting, staying or sleeping for another few days.

- In cases of low dose for large residual tissue patient should be isolated for longer time.

\section{Annexure 10. Care about contaminated items}

3 types of contaminations:
High level:

- In contact with saliva \& urine especially fabric or wipes

- Carefully clean with damp cloth, wash, either hand or washing machine.

- If washing is not practicable put out of way for 6 to 12 weeks.

Medium level:

- Items that have been handled a lot, sneezed or coughed upon

- Decontaminate with damp wipe, wash or put in isolation for 1 month

Low level:

- Items in contact with skin

- Quick wipe over

- To avoid contamination with touch rinse hands with water before touching door handles, key boards, remotes, cell phones etc

\section{Documents and articles consulted:}

1. Alam F, Islam ASMM and Karim MA. Therapy Protocol for Thyroid carcinoma and Thyrotoxicosis with I-131 Adopted in the National Workshop on Management of Thyroid cancer \& Thyrotoxicosis by Nuclear Medicine Technique-A Consensus Report. Bangladesh J Nucl Med 2002;5(2):39-42

2. American thyroid association (ATA) guidelines: Cooper DS, Doherty GM, Haugen BR, Kloos RT et al. Revised American thyroid association management guidelines for patients with thyroid nodules and differentiated thyroid cancer. Thyroid. 2009;19(11):1167-1214.

3. British thyroid association (BTA) guidelines: Perros P, Boelaert K, Colley S, Evans C et al. British thyroid association guidelines for the management of thyroid cancer. Clinical Endocrinology. 2014;81(1):1-122.

4. European thyroid association (ETA) guidelines: Pacini F, Schlumberger M, Dralle H, Elisei R et al. European consensus for the management of patients with differentiated thyroid carcinoma of the follicular epithelium. European Journal of Endocrinology. 2006;154:787-803. 
5. IAEA TECDOC-1608: Nuclear Medicine in thyroid cancer management: A practical approach. IAEA. 2009.

6. Society of Nuclear Medicine (SNM) guidelines: Silberstein EB, Alavi A, Balon HR, Clarke SEM et al. The SNM practice guideline for therapy of thyroid disease with ${ }^{131}$ I. Journal of Nuclear Medicine. 2012;53(10)1633-1651.

7. Seidlin SM, Marinelli LD, Oshry E. Radioactive iodine therapy: Effect on functioning metastases of adenocarcinoma of the thyroid. JAMA. 1946;132:838-847.

8. Luster M, Clarke SE, Dietlein M, Lassmann M et al. Guidelines for radioiodine therapy of differentiated thyroid cancer. Eur J Nucl Med Mol Imaging. 2008;35:1941-1959.

9. Schlumberger M, Berg G, Cohen O, Duntas L et al. Follow-up of low-risk patients with differentiated thyroid carcinoma: a European Perspective. European Journal of Endocrinology. 2004;150:105112.

10.Carballo M, Quiros RM. To treat or not to treat: the role of adjuvant radioiodine therapy in thyroid cancer patients. Journal of Oncology. 2012;2012.

11.Leil Y. Preparation for radioactive iodine therapy in differentiated thyroid cancer patients. Clinical endocrinology (oxf) 2002;57:523-527.

12.Grisby PW, Siegel BA, Bekker S, Clutter WF et al. Preparation of patient with thyroid cancer for ${ }^{131} \mathrm{I}$ scintigraphy of therapy by 1-3 weeks of thyroxine discontinuation. Journal of Nuclear Medicine. 2004;45:567-570.

13. Serhal DI, Nasrallah MP, Arafah BM. Rapid rise in serum Thyrotropin concentrations after thyroidectomy or withdrawal of suppressive thyroxine therapy in preparation for radioactive iodine administration to patients with differentiated thyroid cancer. Journal of Clinical Endocrinology Metabolism. 2004;89:3285-3289.

14.Nurun Nahar, Sadia Sultana, Lutfun Nisa, Gita Rani Howladar, AHM Shahidul Islam, Faridul Alam. Importance of early TSH estimation after total thyroidectomy for well differentiated thyroid carcinoma. Bangladesh Journal of Nuclear Medicine. 2008;11(2):123-126.Kuijt WJ, Huang SA. Children with differentiated thyroid cancer achieve adequate hyperthyrotropinemia within 14 days of levothyroxine withdrawal. Journal of Clinical Endocrinology Metabolism. 2005;90:6123-6125.

15. Sultana S, Nahar N, Haque FS, Alam F, Nisa L, Rahman T. Can withdrawal of levothyroxine for three weeks achieve optimum TSH level required for whole body iodine scan in patients with differentiated thyroid carcinoma? Bangladesh Journal of Nuclear Medicine. 2008;11(2):127-130.

16. Heemstra KA, Liu YY, Stokkel M, Kievit J et al. Serum thyroglobulin concentrations predict diseasefree remission and death in differentiated thyroid carcinoma. Clinical Endocrinology. 2007;66:58-64.

17. Sultana S, Nahar N, Haque FS, Alam F, Rahman MA. Outcome in patients with differentiated thyroid carcinoma with detectable serum thyroglobulin associated with negative diagnostic whole body scan. Bangladesh Journal of Nuclear Medicine. 2007;10(1):21-26.

18.Jarzab B, Handkiewicz-Junak D, Wloch J. Juvenile differentiated thyroid carcinoma and the role of radioiodine in its treatment: a qualitative review. Endocrine related Cancer. 2005;12:773-803.

19. Chianelli M, Todino V, Graziano F, Panunzi C et al. Low dose (2.0GBq; $54 \mathrm{mCi})$ radioiodine postsurgical remnant ablation in thyroid cancer: comparison between hormone withdrawal and use of rhTSH in low risk patients. European Journal of Endocrinology. 2009;160:431-436.

20.Lewis BD, Hat ID, Charboneau JW, Mclver B et al. Percutaneous ethanol injection for treatment of cervical lymph node metastases in patients with papillary thyroid carcinoma. American Journal of Roentgenology. 2002;178:699-704.

21.Precaution after doses of radioactive iodine (ARI) for thyroid cancer. http://www.thyroidcancercanada. org/userfiles/files/RAI_precautions_Ian_Adam_201 0 revised.pdf

22. Radiation protection following radioactive iodine (I131) ablation treatment http://www.hey.nhs.uk /Downloads/Patient\%20Leaflets/Nuclear\%20Medici ne/Radiation\%20Protection\%20following\%20Iodine $-131 \% 20 \mathrm{I}-131 \% 20$ Ablation\%20Treatment.pdf

23.Precaution after out-patient radioactive iodine (I131) therapy http://www.stjoes.ca/patientsvisitors/patienteducation/fj/PD\%206177\%20I131Ra dioactiveIodineTherapySJH-trh.pdf 